\title{
High dose dipyridamole as a pharmacological stress test during cardiac catheterisation in patients with coronary artery disease
}

\author{
Philipp Wagdi, Urs Kaufmann, Martin Fluri, Bernhard Meier
}

\begin{abstract}
Aim-To validate dipyridamole as a pharmacological stress test during cardiac catheterisation, allowing both functional and morphological estimation of stenosis severity.

Methods-The study encompassed 74 patients: 62 patients with significant coronary artery disease (age 61 (SD 8) years; seven women, 55 men) and 12 controls. Regional wall motion, left ventricular ejection fraction and end diastolic pressure were analysed in the resting state and after high dose intravenous dipyridamole. Patients were subdivided into four groups: group I $(n=32,43 \%)$ had stopped all anti-ischaemic treatment for $>24 \mathrm{~h}$, group II $(\mathrm{n}=14,19 \%)$ was under treatment, group III $(n=16,22 \%)$ had significant coronary artery disease only in regions with regional wall motion abnormalities at rest, and group IV consisted of 12 control patients (16\%) with no significant coronary artery disease (age 62 (8) years, three women, nine men).

Results-The sensitivity of dipyridamole testing in patients with coronary artery disease was poor. The best sensitivity was obtained with regional wall motion analysis $(26 / 62=42 \%)$ and with global left ventricular ejection fraction $(25 / 62=40 \%)$. Specificity was $100 \%$ for regional wall motion and $100 \%$ for ejection fraction. Calculated positive and negative predictive values for regional wall motion were $100 \%$ and $63 \%$, respectively.

Conclusions-Although safe, handy, and inexpensive, dipyridamole is not an adequate pharmacological stress test during cardiac catheterisation because of its low sensitivity.
\end{abstract}

(Heart 1996;75:247-251)

Keywords: dipyridamole; stress testing; ischaemic heart disease; coronary stenosis severity

Department of Cardiology, University Hospital, Bern, Switzerland P Wagdi

U Kaufmann

M Fluri

B Meier

Correspondence to: P Wagdi MD, Cardiac Catheterisation Laboratory Department of Cardiology, Kantonsspital, 5001 Aarau, Kantonsspital
Switzerland.

Accepted for publication 25 September 1995 These agents have been applied in echocardiography, ${ }^{3}$ radionuclide ventriculography, ${ }^{4}$ and perfusion imaging. ${ }^{1}$ After initially enthusiastic reports, the use of dipyridamole in functional imaging has recently been put into perspec- tive, ${ }^{56}$ although it continues to be promoted. ${ }^{7}$ The demonstration of regional ischaemia is an important diagnostic adjunct to cardiac catheterisation. Stenotic coronary lesions are increasingly approached interventionally immediately after the diagnostic study, ${ }^{8}$ obviating the need for recatheterisation and thus lessening patient discomfort and morbidity, and contributing to cost containment. Exercise testing or pacing for ischaemia induction in the catheterisation laboratory ${ }^{9}$ has been used but both are impractical. A sensitive, specific, and safe non-exercise stress test that could be applied in the wake of cardiac catheterisation would help in rapid and adequate decision making and, in selected cases, obviate the need for additional diagnostic tests. Cost containment, one of the major issues in today's health care, will also affect cardiac imaging. ${ }^{10}$ Because coronary angiography is likely to remain the diagnostic cornerstone in managing coronary artery disease, combining it with an accurate, inexpensive, and well tolerated functional test would be particularly attractive. The aim of this study was to determine whether dipyridamole met the criteria for diagnosing ischaemia during cardiac catheterisation and whether its use in the setting of stress echocardiography was valid, in the light of the fact that both echocardiography and radionuclide ventriculography have limitations. ${ }^{11}$

\section{Methods}

One hundred and twenty consecutive patients with known or suspected coronary artery disease were enrolled. They had been referred for diagnostic cardiac catheterisation including coronary angiography. Exclusion criteria were obstructive lung disease, atrial fibrillation, frequent ventricular or supraventricular ectopy, valvar heart disease, left heart failure, more than two previous myocardial infarcts, or unstable angina pectoris. Fourteen patients refused to participate. The protocol could not be performed in 21 patients because of constraints involving time and laboratory personnel. Five studies were disregarded because of non-sustained ventricular tachycardia during ventriculography, leaving no nonpostextrasystolic beat for analysis of ejection fraction and regional wall motion. Six patients with no coronary artery disease were excluded from the control group because they had dilated cardiomyopathy. The remaining 74 patients were subjected to the protocol and analysed. In most patients a 12-lead ECG 
Table 1 Patient characteristics

\begin{tabular}{|c|c|c|c|c|}
\hline & $\begin{array}{l}\text { Group I } \\
(n=32)\end{array}$ & $\begin{array}{l}\text { Group II } \\
(n=14)\end{array}$ & $\begin{array}{l}\text { Group III } \\
(n=16)\end{array}$ & $\begin{array}{l}\text { Group IV } \\
(n=12)\end{array}$ \\
\hline Age, years $(\mathrm{SD}) /$ gender & $60(7) / 4 \mathrm{~F}$ & $62(11) / 2 \mathrm{~F}$ & $62(8) / 1 F$ & $62(8) / 3 F$ \\
\hline $\begin{array}{l}\text { Diagnosis: } \\
\text { One vessel disease } \\
\text { Two vessel disease } \\
\text { Three vessel disease } \\
\text { Extracardial chest pain } \\
\text { Hypertensive heart disease }\end{array}$ & $\begin{array}{l}6 \\
11 \\
15^{\star} \\
-\end{array}$ & $\begin{array}{l}8 \\
3 \\
3^{\star} \\
- \\
-\end{array}$ & $\begin{array}{l}10 \\
4 \\
2^{\star} \\
- \\
-\end{array}$ & $\begin{array}{l}- \\
\overline{7} \\
5\end{array}$ \\
\hline $\begin{array}{l}\text { Presenting symptoms: } \\
\text { Typical angina pectoris } \\
\text { Dyspnoea } \\
\text { Ventricular extrasytoles } \\
\text { Atypical chest pain } \\
\text { None }\end{array}$ & $\begin{array}{r}30 \\
1 \\
1 \\
0 \\
0\end{array}$ & $\begin{array}{r}13 \\
0 \\
0 \\
0 \\
1\end{array}$ & $\begin{array}{r}12 \\
0 \\
0 \\
1 \\
3\end{array}$ & $\begin{array}{l}8 \\
1 \\
0 \\
2 \\
2\end{array}$ \\
\hline $\begin{array}{l}\text { Stress test before catheterisatic } \\
\text { Positive } \\
\text { Equivocal } \\
\text { Negative } \\
\text { Not done }\end{array}$ & $\begin{array}{r}23 \\
4 \\
2 \\
3\end{array}$ & $\begin{array}{r}11 \\
2 \\
1 \\
0\end{array}$ & $\begin{array}{r}11 \\
1 \\
1 \\
3\end{array}$ & $\begin{array}{l}8 \\
0 \\
2 \\
2\end{array}$ \\
\hline $\begin{array}{l}\text { Side effects of dipyridamole }+ \text { : } \\
\text { Angina pectoris } \\
\text { Dizziness } \\
\text { Headache } \\
\text { None }\end{array}$ & $\begin{array}{r}15 \\
15 \\
5 \\
11\end{array}$ & $\begin{array}{r}5 \\
1 \\
2 \\
10\end{array}$ & $\begin{array}{l}9 \\
3 \\
2 \\
5\end{array}$ & $\begin{array}{l}7 \\
5 \\
0 \\
4\end{array}$ \\
\hline
\end{tabular}

$\star$ Three vessel disease in group I $v$ groups II and III; $\mathrm{P}=0.01$.

tMultiple side effects were sometimes reported by the patients so that the numbers do not add up in each group.

bicycle stress test had been done before the study (table 1) and all of them had been fasting overnight before catheterisation.

The patients were subdivided into four groups (table 1): group I ( $\mathrm{n}=32,43 \%)$ comprised patients who had stopped all antianginal medication for at least $24 \mathrm{~h}$ before cardiac catheterisation; group II ( $\mathrm{n}=14,19 \%)$ comprised patients who had continued medication either inadvertently or because of therapeutic considerations; group III $(n=16,22 \%)$ comprised patients in whom the diseased vessel(s) exclusively supplied a territory with regional wall motion abnormalities at rest; group IV (n $=12,16 \%$ ) comprised patients without significant coronary artery disease. Thirty one percent (10/32) of group I, 29\% (4/14) of group II, and $100 \%(16 / 16)$ of group III patients had a previous myocardial infarct. Of the 62 patients, $22(35 \%)$ had at least one completely occluded artery and collaterals supplying its territory, five $(8 \%)$ had an occluded artery and no collaterals, and one had a critically stenosed and collateralised artery. All percentage figures are rounded up.

\section{STUDY PROTOCOL}

The study protocol was approved by the institutional review board and all patients gave informed consent. Catheterisation was performed through a $5 \mathrm{~F}$ sheath. A pigtail $5 \mathrm{~F}$ catheter was used for ventriculography and pressure measurements. The following parameters were recorded after four consecutive sinus beats during each sequence: left ventricular systolic and end diastolic pressures, heart rate and a three-lead ECG tracing. First baseline data were recorded. Biplane contrast ventriculography with $50 \mathrm{ml}$ of ioversol $(12 \mathrm{ml} / \mathrm{s})$ was then performed in standard right anterior oblique $30^{\circ}$ and left anterior oblique $60^{\circ}$ views. After ventriculography, pressure readings were repeated. Dipyridamole was infused in a dose of $0.84 \mathrm{mg} / \mathrm{kg}$ body weight over 6 min. After a 2 min pause to attain a steady state, the haemodynamic indices were mea- sured again, followed within $1 \mathrm{~min}$ by the second ventriculography and final measurement of haemodynamic indices. Finally, $125 \mathrm{mg}$ of aminophylline were given to offset any side effects. Coronary angiography was then performed.

The global left ventricular ejection fraction was calculated by semiautomated software. Biplane regional wall motion and coronary anatomy were visually assessed independently by two observers. Interobserver variability, resolved by consensus, was $8 \%$ (20 segments disagreed upon out of 248 ventriculograms, that is 62 patients with coronary heart disease, each having a right anterior oblique and a left anterior oblique sequence both at rest and under dipyridamole) and was due exclusively to differences in assessing the grade of hypokinesia as being mild or severe.

\section{DEFINITIONS}

Coronary artery disease was judged significant if one or more $>50 \%$ diameter stenoses were found to be present in at least two views, by consensus of at least four observers during routine case review independently of the study. Regional wall motion was described using a numerical score: 1, normal wall motion; 0 , mild to moderate hypokinesia; -1 , severe hypokinesia; -2 , akinesia; -3 , dyskinesia. Regional wall segments were defined as anterobasal, anterior, apical, inferior, and posterobasal in the right anterior oblique view, and septal, apical, and posterolateral in the left anterior oblique view. Points of regional wall

Table 2 Double product after ventriculography at rest and after dipyridamole, values are means (SD)

\begin{tabular}{llc}
\hline & Rest & Dipyridamole \\
\hline Group I (n=32) & $8919(2020)$ & $10553(2108)^{\star}$ \\
Group II $(\mathrm{n}=14)$ & $7926(2101)$ & $8723(2026) \mathrm{NS}$ \\
Group III $(\mathrm{n}=16)$ & $8212(1613)$ & $10837(2064)^{\star}$ \\
Group IV $(\mathrm{n}=12)$ & $9605(1847)$ & $11287(1965)^{\star}$ \\
\hline
\end{tabular}

Double product $=$ heart rate systolic blood pressure in ${ }_{\star} \mathrm{m}<0.001$. 
Table 3 Variables recorded at rest $(R)$ and under dipyridamole (D). Values are means (SD)

\begin{tabular}{lllllll}
\hline & & $\begin{array}{c}\text { All CAD } \\
(n=32)\end{array}$ & $\begin{array}{l}\text { Group I } \\
(n=32)\end{array}$ & $\begin{array}{l}\text { Group II } \\
(n=14)\end{array}$ & $\begin{array}{l}\text { Group III } \\
(n=16)\end{array}$ & $\begin{array}{l}\text { Group IV } \\
(n=12)\end{array}$ \\
\hline EF (\%) & $\mathrm{R}$ & $60(15)$ & $63(17)$ & $64(12)$ & $52(11)$ & $71(6)$ \\
& $\mathrm{D}$ & $61(17) \mathrm{NS}$ & $64(19) \mathrm{NS}$ & $63(14) \mathrm{NS}$ & $54(13) \mathrm{NS}$ & $76(7)^{\star}$ \\
RWM & $\mathrm{R}$ & $3 \cdot 6(3 \cdot 8)$ & $4(3 \cdot 9)$ & $4 \cdot 5(3)$ & $1.9(3 \cdot 8)$ & 7 \\
& $\mathrm{D}$ & $3(4 \cdot 1)^{\star}$ & $3(4 \cdot 5)^{\star}$ & $3 \cdot 9(3 \cdot 2) \mathrm{NS}$ & $1.9(3 \cdot 8) \mathrm{NS}$ & 7 \\
LVEDP & $\mathrm{R}$ & $18(8)$ & $18(7)$ & $17(7)$ & $18(9)$ & $16(6)$ \\
& $\mathrm{D}$ & $20(9)^{\star}$ & $23(7)^{\star}$ & $18(10) \mathrm{NS}$ & $20(10)^{\star}$ & $20(6)^{\star}$ \\
\hline
\end{tabular}

$\mathrm{CAD}$, coronary artery disease; $\mathrm{EF}$, left ventricular ejection fraction; LVEDP, left ventricular end diastolic pressure in mm $\mathrm{Hg}$; RWM, regional wall motion score.

${ }^{\star} \mathrm{P}<0.05$. Significance refers to variable at rest $v$ dipyridamole within a group. No comparison is made between groups.

Table 4 Percentage of positive ischaemic responses using all criteria

\begin{tabular}{lllll}
\hline & $\begin{array}{l}\text { Group I } \\
(n=32)\end{array}$ & $\begin{array}{l}\text { Group II } \\
(n=14)\end{array}$ & $\begin{array}{l}\text { Group III } \\
(n=16)\end{array}$ & $\begin{array}{l}\text { Group IV } \\
(n=12)\end{array}$ \\
\hline EF & $13(41 \%)$ & $4(29 \%)$ & $8(50 \%)$ & $0(0 \%)$ \\
WM & $13(41 \%)$ & $7(50 \%)$ & $6(38 \%)$ & $0(0 \%)$ \\
LVEDP & $15(47 \%)$ & $6(43 \%)$ & $4(25 \%)$ & $5(42 \%)$ \\
\hline
\end{tabular}

EF, left ventricular ejection fraction; WM, wall motion; LVEDP, left ventricular end diastolic pressure. Criteria of positivity for ischaemia: increase of $1 \mathrm{SD}$ or more (LVEDP), failure to rise $5 \%$ or more or fall of $\mathrm{EF}$ in patients with subnormal EF at rest, fall of $\mathrm{EF}>5 \%$ in patients with normal EF, and any decrease in wall motion.

motion were added, yielding a wall motion score. The latter was not meant to be a quantitative, but rather a qualitative descriptor.

Ischaemia under dipyridamole was defined as: (a) wall motion worsening by 1 or more units (either on total score or when contractility of a segment worsened without the total score falling numerically, as happened when another segment's contractility improved); (b) fall or absence of rise in global ejection fraction when ejection fraction was reduced $(<55 \%)$ at rest, or a fall of $>5 \%$ when ejection fraction was normal at rest.

\section{STATISTICAL ANALYSIS}

Significance between variables at rest and under dipyridamole in the same group was computed using a two tailed paired $t$ test. Significance was set at $\mathrm{P}<0.05$. Positive predictive value was computed as true posi-
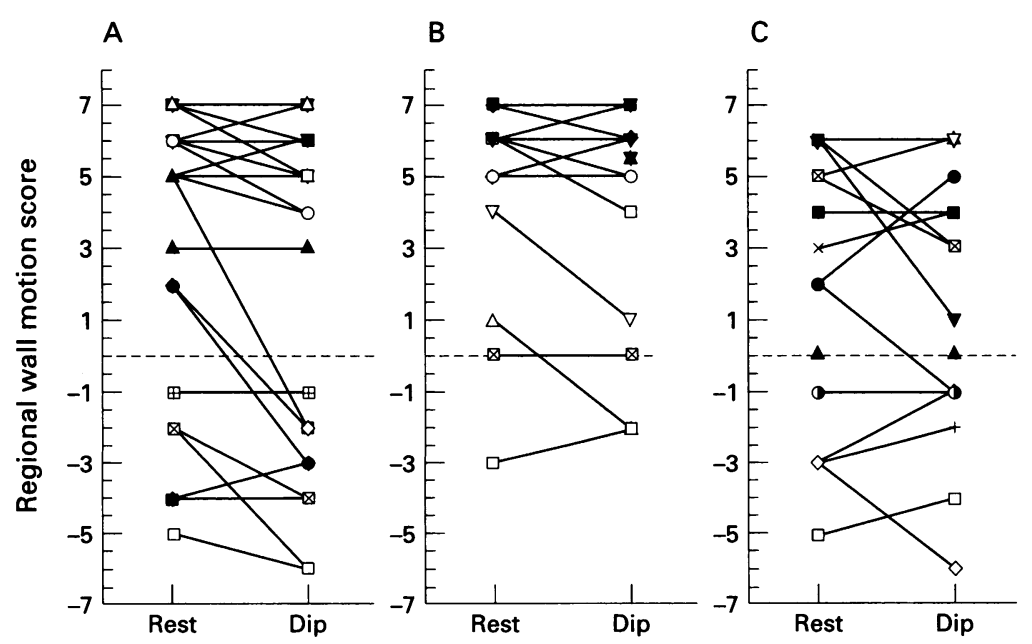

Regional wall motion in (A) group I patients, (B) group II patients, and (C) group III patients at rest and after dipyridamole (Dip). Some patients overlap, eg, in eight patients in group I a baseline WMS of 7 remained unchanged under dipyridamole. WMS, wall motion score. tives/true positives + false positives. Negative predictive value was calculated as true negatives/true negatives + false negatives.

\section{Results}

Although significant coronary artery disease was defined as $>50 \%$ diameter stenosis, only two patients in group I and one patient in group II showed coronary artery disease with $<70 \%$ diameter stenosis of one or more vessels, so that in 59 patients the coronary artery disease was severe. The double product (heart rate $\times$ blood pressure) change from the resting condition to dipyridamole stress is shown in table 2 ; the change is significant except for patients under antianginal treatment (group II). The change in left ventricular ejection fraction was significant only in group IV. Table 3 shows the change in measured variables before and after dipyridamole. The left ventricular end diastolic pressure rose significantly except for the group of patients under medication and yielded a high overall positive response (table 4). Wall motion changes from baseline under dipyridamole are shown in the figure. In patients with three vessel disease and no antianginal treatment, a total of $41 \%$ (in group II 50\%) of positive responses was attained. Importantly, among 12 group I patients who had no wall motion abnormalities at rest, eight $(67 \%)$ kept a maximum wall motion score under dipyridamole.

Using significant angiographic coronary artery disease as a reference and dipyridamole induced regional wall abnormality as a criterion, true positives occurred in $42 \%$ of the entire group, and false negatives in $58 \%$. For group I, there were $41 \%$ true positives and for group II $50 \%$ (table 4 ), with $59 \%$ and $50 \%$ false negatives respectively. Calculation of true positives and false negatives for group III may not be accurate because in an infarcted area there may not be any myocardium left to demonstrate an ischaemic response. In group IV there were no false positives and $100 \%$ true negatives. The positive predictive value of regional wall motion worsening under dipyridamole was $100 \%$, the negative predictive value was $63 \%$.

A relatively high percentage of patients were symptomatic after dipyridamole (table 1). Globally, 38/62 patients in groups I-III (61\%) and $8 / 12$ patients in group IV $(67 \%)$ complained of one or more symptoms. All side effects were promptly reversed by aminophylline after all data had been gathered. 


\section{Discussion}

STUDY LIMITATIONS

The study was not blinded. Alternating the stress and baseline measurements was not done to avoid unduly prolonging the catheterisation time. If dipyridamole had been infused first in some patients, at least half an hour would have been required for the haemodynamic effects of the drug to wear off before performing resting measurements. Giving the antidote aminophylline does not restore baseline conditions, as it exerts significant changes on cardiac performance itself. ${ }^{12}$ On the one hand, the injected volume load during resting state ventriculography would be expected to increase preload and thus have a positive effect on ejection fraction except in the severely depressed ventricle. On the other hand, even non-ionic contrast media are known to exert a mild depressant effect on left ventricular function, so that the net effect of this sequence of examination would be unpredictable. ${ }^{13-15}$

The patients examined were referred to the hospital for cardiac catheterisation. Most of them had significant coronary artery disease, and some were on antianginal treatment, which may have blunted the ischaemic effect of dipyridamole. Yet of the groups with the largest experience with dipyridamole stress testing, one reported a sensitivity of $65 \%$ for patients on antianginal treatment. ${ }^{16} \mathrm{We}$ could not confirm this result in our group II patients, only $50 \%$ of whom had a positive result. Furthermore, a positive bicycle stress test under antianginal treatment had been done in most patients in our series before cardiac catheterisation (table 1). Patients with myocardial infarction form an important subgroup requiring functional and prognostic assessment $^{7}$; many of these patients have wall motion abnormalities exclusively in the territory of the infarct arteries (our group III). In this subgroup again, analysis of wall motion had a low yield of positive results, although $50 \%$ showed a fall in global left ventricular ejection fraction.

\section{LEFT VENTRICULAR EJECTION FRACTION AND REGIONAL WALL MOTION}

Left ventricular ejection fraction and particularly regional wall motion are the classical variables used in functional stress imaging. Regional wall motion was the overall most sensitive index except in group III patients. Although many patients showed a hypercontractile state under dipyridamole, this was noted as normokinesia when scoring wall motion. The reason is that if a hyperkinesia score of 2 was attributed in these situations a falsely high wall motion score would result (for example, a patient with normokinesia of all segments at rest, severe hypokinesia in two segments, and hyperkinesia in the others under diypridamole would have a score of 7 at rest and 8 under dipyridamole, thus being falsely counted as "negative" and further lowering the sensitivity of the test). Three patients in groups I and II and six patients in group III (see the figure) showed an increase in total wall motion score under dipyridamole.
Whether this represents true "recruitment" of stunned or hibernating myocardium, or whether these segments were just passively pulled along, giving the false impression of improved contractility (see below), is unclear. This phenomenon occurred much more often in group III.

\section{LEFT VENTRICULAR END DIASTOLIC PRESSURE}

Had it not been for a high false positive result in group IV, left ventricular end diastolic pressure would have compared favourably with regional wall motion. Several haemodynamic factors in this study, however, limit its value in assessing ischaemia, namely the repeated volume challenge with contrast medium, and the relatively high age of the patients with decreased left ventricular compliance even in the absence of ischaemia (table 1). Globally, the left ventricular end diastolic pressure rose in all groups except for group II. This may point to a favourable effect of antianginal treatment on diastolic function.

Dipyridamole stress results compare unfavourably with the $45 / 59$ positive bicycle stress tests $(76 \%)$ in our patients (table 1$)$. On the other hand $38 \%$ of group IV patients had a false positive bicycle stress test. These results reflect tests in a selected population with a rather high positive pretest probability (hypertensive heart disease, old age). The reason for the lower sensitivity of dipyridamole may be the fact that to produce regional wall motion disturbances a test must induce subendocardial ischaemia. ${ }^{17}$ In patients aged $50-60$ years undergoing ergometry, the double product had already risen to 18300 units at $50 \mathrm{~W},{ }^{18}$ showing that although the pressure product rises significantly under dipyridamole, the rise is well below that attained by physical exercise (table 2).

In patients with severe three vessel disease and impaired left ventricular function the need for bypass surgery is usually obvious. In patients with preserved function, there should be proof of reversible ischaemia. Likewise, in most patients with severe one or two vessel disease, percutaneous transluminal coronary angioplasty (PTCA) is usually indicated. On the other hand, it is important to identify ischaemia in patients with borderline one and two vessel disease. Because PTCA carries about a $5 \%$ risk of acute occlusion and a $30-40 \%$ risk of restenosis, it is controversial whether angioplasty of moderate lesions is of benefit when no ischaemia of the respective territory can be demonstrated. Even in patients with coronary artery disease exclusively in a territory with regional wall motion abnormalities at rest and in those under antianginal treatment, demonstration of functional impairment under stress is of prognostic importance. Dipyridamole has not yet been conclusively shown to detect hibernating or stunned myocardium by recruitment of still viable myocardial fibres.

\section{COMPARISON WITH ECHOCARDIOGRAPHY}

Wall motion during contrast ventriculography is assessed in two planes (right and left ante- 
rior oblique), in contrast to the four planes used in echocardiography (parasternal short and long axis, apical two and four chamber views). One may argue that echocardiography allows a more detailed analysis of wall motion, explaining the better results reported. On the one hand, the different regions definitely overlap in echocardiography, so the regions analysed are not necessarily more than those viewed by ventriculography. Most of the myocardium is displayed by biplane ventriculography. Wall motion changes of any significance are detected by ventriculography, which definitely has the edge over echocardiography in terms of resolution. With the latter method, regional wall motion cannot be adequately studied in at least $20 \%$ of patients because of technical limitations, especially in the apical and anterolateral segments. In echocardiographic studies real time data are continuously sampled during and up to 10 minutes after infusion for regional wall motion abnormalities. The lack of temporal sampling of wall motion abnormality may be a limitation of the present study, the left ventriculogram giving a "snapshot" of wall motion and lacking the repetitive assessment provided by echocardiography. On the other hand, a steady state is reached two to three minutes after termination of infusion, ${ }^{3}$ so at the time of ventriculography it may not be possible in some patients to detect signs of transient ischaemia that occurred before or after ventriculographythough this is somewhat unlikely.

The examination and quantification of regional wall motion is fraught with pitfalls in all methods of assessing regional wall motion. Even with experienced observers, the effect of tethering by neighbouring muscle and cardiac translation can be confusing. The reason for the discrepancy between our results and those reported by the groups promoting dipyridamole echocardiography are thus unlikely to lie in pitfalls of regional wall motion analysis. It is noteworthy that other groups have reported relatively low sensitivities with dipyridamole $^{6}$ and adenosine. ${ }^{2}$ The explanation may rather lie in the fact that dipyridamole does not produce subendocardial ischaemia. ${ }^{17}$ It produces flow heterogeneity, ${ }^{3}$ and is thus an inadequate pharmacological stressor for functional imaging, but a good tool when used in conjunction with perfusion studies. ${ }^{12}$

SIDE EFFECTS

Our patients reported substantially more side effects from dipyridamole than are acknowledged in published reports. ${ }^{3}$ The reason for this may be that the test was performed in a group with more severe disease than in other studies. Yet a high percentage of side effects was also noticed in patients of the control group.

\section{CONCLUSIONS}

Although dipyridamole stress ventriculography during cardiac catheterisation is a handy and safe method, it had too low sensitivity in our experience for the assessment of functional severity of coronary artery stenosis when compared to bicycle stress testing. The data presented support arguments against the widespread use of dipyridamole echocardiography as a pharmacological stress test.

1 Taillefer R. Technetium-99m Sestamibi myocardial imaging: same-day rest-stress studies and dipyridamole. $A m \mathcal{F}$ Cardicl 1990;66:80-84E.

2 Marwick T, Willemart B, D'Hont A, et al. Selection of the optimal nonexercise stress test for the evaluation of ischemic regional myocardial dysfunction and malperfusion. Circulation 1993;87:345-54

3 Picano E. Dipyridamole-echocardiography test: historical background and physiologic basis. Eur Heart $\mathcal{f} 1989 ; 10$ 365-76.

4 Cates CU, Kronenberg MW, Collins HW, Sandler MP Dipyridamole radionuclide ventriculography: A test with high specificity for severe coronary artery disease. $\mathcal{F} A m$ Coll Cardiol 1989;13:841-51.

5 Harris D, Taylor D, Condon B, Ackery D, Conway N. Myocardial imaging with dipyridamole: Comparison of the sensitivity and specificity of ${ }^{201}$ TI versus MUGA. Eur $\mathcal{F}$ Nucl Med 1982;7:1-5.

6 Mazeika P, Nihoyannopoulos P, Joshi J, Oakley C. Uses and limitations of high dose dipyridamole stress echocardiography for evaluation of coronary artery disease. $B r$ diography for evaluation

7 Picano E, Landi P, Bolognese L, et al. Prognostic value of dipyridamole echocardiography early after uncomplicated myocardial infarction: A large scale multicente trial. Am f Med 1993;95:608-18.

8 Moles VP, Meier B, Pande AK. PTCA at first sight: angioplasty based on video only. $\mathcal{F}$ Invas Cardiol 1992;4:344-8

9 Aroesty JM, Mc Kay RG, Heller GV, Royal HD, Als AV Grossmann $W$. Simultaneous assessment of left ventricular systolic and diastolic dysfunction during pacinginduced ischemia. Circulation 1985;71:889-900.

10 Vogel RA. The impact of health care reform on cardiac imaging. Curr Opin Cardiol 1993;8:975-7.

11 Crawford MH, Petru MA, Amon KW, Sorensen SG, Vance WS. Comparative value of 2-dimensional echocardiography and radionuclide ventriculography for quantidiography and radionuclide ventriculography for quanttating changes in left ventricular performance durin exercise limited by angina pectoris. Am f Cardiol 1984, 53:42-6.

12 Ogilvie RI, Fernandez PG, Winsberg F. Cardiovascular response to increasing theophylline concentrations. Eur $\mathcal{F}$ Clin Pharmacol 1977;12:409-14.

13 Aguirre FV, Pedersen W, Castello R, et al. The effects of high (sodium meglumine diatrizoate, Renografin-76) and low osmolar (sodium meglumine ioxaglate, Hexabrix) radiographic contrast media on diastolic function during left ventriculography in patients. Am Heart $\mathcal{F} 1991 ; 121$ 848-57.

14 Ritchie JL, Nissen SE, Douglas JS, et al. Use of nonionic or low osmolar contrast agents in cardiovascular procedures. F Am Coll Cardiol 1993;21:269-73.

15 Gerber KH, Higgins CB, Yuh YS, Koziol JA. Regional myocardial hemodynamic and metabolic effects of ionic and nonionic contrast media in normal and ischemic states. Circulation 1982;65:1307-14.

16 Lattanzi F, Picano E, Bolognese L, et al. Inhibition of dipyridamole-induced ischemia by antianginal therapy in humans: correlation with exercise electrocardiography. Circulation $1991 ; 83: 1256-62$.

17 Fung AY, Gallagher KP, Buda AJ. The physiological basis of dobutamine as compared with dipyridamole stress interdobutamine as compared with dipyridamole stress interventions in the assessment
Circulation 1987;76:943-51.

18 Fronek A, Hofbauer KG, Kalusche D, et al. Heart rate. In Lentner C, ed. Geigy scientific tables. Heart and circulation. Basel: Ciba Geigy, 1990:16. 